\title{
Conhecimento e opinião de alunos da educação básica sobre Infecções Sexualmente Transmissíveis (IST)
}

\author{
Knowledge and opinion of basic education students on Sexually Transmitted Infections
}

\section{Conocimiento y opinión de alumnos de educación básica sobre Infecciones Sexualmente Transmisibles (IST)}

Everton Luis Freitas Wanzeler ${ }^{1 *}$, Jamille Marcelle Ribeiro Costa ${ }^{1}$, Ailini Danielle Ribeiro Costa ${ }^{1}$, Geovanna Carey Brabo da Silva ${ }^{1}$, Taize Vieira da Cruz ${ }^{1}$, Antonia Tamires Penha da Costa ${ }^{1}$, Tamires

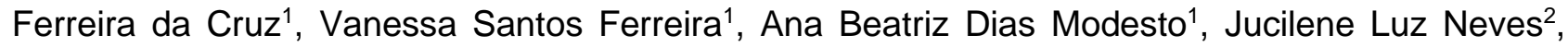
Bárbara Melissa Silva Pereira1, Gabriel Vinicius Reis de Queiroz¹.

\section{RESUMO}

Objetivo: Verificar o conhecimento e opinião de estudantes da educação básica sobre infecções sexualmente transmissíveis numa escola estadual localizada em um município do estado do Pará. Métodos: Estudo transversal descritivo, quantitativo com 300 alunos do Ensino Médio, realizado numa instituição de ensino pública em um município do estado do Pará, no período de setembro a outubro de 2018. Resultados: A média de idade dos pesquisados foi 17 anos e $69 \%$ dos estudantes foram do gênero feminino. Dos entrevistados $186(62 \%)$ estudantes informaram já terem iniciado a vida sexual. Sobre o conhecimento a respeito das doenças que podem ser transmitidas durante a relação sexual desprotegida 252 (82\%) estudantes responderam que se transmitem doenças infectocontagiosas sistêmicas sem o uso de preservativo. Conclusão: Observa-se nesta reflexão que as situações de vulnerabilidade dos adolescentes a IST relacionam-se com a não compreensão do uso de preservativos, baixa escolaridade, conhecimento e informação deficientes, fatores culturais e a falta de orientações.

Palavras-chave: Infecções, Conhecimento, Prevenção.

\begin{abstract}
Objective: To verify the knowledge and opinion of basic education students about sexually transmitted infections in a state school located in a municipality in the state of Pará. Methods: A descriptive, quantitative cross-sectional study with 300 high school students, carried out at a public school in Barcarena / PA, from September to October 2018. Results: The average age of the respondents was 17 years and $69 \%$ students were female. Of the interviewees, $186(62 \%)$ students reported having started their sex life. About the knowledge about diseases that can be transmitted during unprotected sexual intercourse $252(82.0 \%)$ students answered that infectious contagious diseases are transmitted without the use of condoms. Conclusion: It is observed in this reflection that the situations of vulnerability of adolescents to STI arerelated to the lack of understanding of the use of condoms, low educational level, poor knowledge and information, cultural factors and lack of guidelines.
\end{abstract}

Key words: Infections, Knowledge, Prevention.

\section{RESUMEN}

Objetivo: Verificar el conocimiento y la opinión de los estudiantes de la educación básica sobre infecciones de transmisión sexual en una escuela estatal ubicada en un municipio del estado de Pará. Métodos: Estudio

\footnotetext{
${ }^{1}$ Universidade da Amazônia, Belém - PA. *E-mail: evertonwanzeler@hotmail.com

${ }^{2}$ Centro Universitário Metropolitano da Amazônia, Belém - PA.
} 
transversal descriptivo, cuantitativo con 300 alumnos de la Enseñanza Media, realizado en una institución de educación pública en un municipio del estado de Pará, en el período de septiembre a octubre de 2018. Resultados: El promedio de edad de los encuestados fue 17 años y 69, \% de los estudiantes fueron del género femenino. De los entrevistados $186(62 \%)$ estudiantes informaron ya haber iniciado la vida sexual. Sobre el conocimiento acerca de las enfermedades que pueden ser transmitidas durante la relación sexual desprotegida $252(82,0 \%)$ estudiantes respondieron que se transmiten enfermedades infectocontagiosas sistémicas sin el uso de preservativo. Conclusión: Se observa en esta reflexión que las situaciones de vulnerabilidad de los adolescentes a la IST se relacionan con la no comprensión del uso de preservativos, baja escolaridad, conocimiento e información deficientes, factores culturales y la falta de orientaciones.

Palabras clave: Infeccione, Conocimiento, Prevención.

\section{INTRODUÇÃO}

As Infecções Sexualmente Transmissíveis (IST) estão entre os problemas de saúde pública mais comuns mundialmente, sendo consideradas o principal fator facilitador da transmissão sexual do Vírus da Imunodeficiência Humana (HIV) (GOMES NCRC, et al., 2017).

Os adolescentes constituem um grupo que vem, nos últimos anos, exibindo importante suscetibilidade e exposição a situações de riscos, sendo a infecção pelo HIV uma importante forma de contaminação quando 0 ato sexual é desprotegido, demonstrando a necessidade de uma atenção integral a este grupo (SILVA JLGD, et al.,2016).

A média de idade da primeira relação sexual com penetração, no Brasil, estimada é de 14 anos e quatro meses para o sexo masculino e de 15 anos e dois meses para o feminino (FERREIRA J, 2015). Ao mesmo tempo, a liberação dos costumes e a erotização da mídia através da letra de músicas, vêm estimulando uma iniciação sexual cada vez mais precoce, colaborando para o aumento das chances de jovens desenvolverem Infecções Sexualmente Transmissíveis (OLIVEIRA SHS, et al., 2015).

No entanto, estima-se que, a cada ano, um contingente de quatro milhões de jovens torna-se ativos sexualmente no Brasil. $O$ início precoce da vida sexual pode ser considerado um agravante para 0 comportamento de risco frente as IST. O número elevado de ocorrências de gravidez na adolescência em jovens entre 10 e 19 anos, somado ao aumento da ocorrência de IST e à intensificação do consumo de drogas, ajuda-nos a entender melhor porque os jovens brasileiros são, cada vez em maior número, vulneráveis à infecção pelo HIV (OLIVEIRA SHS, et al., 2015; BATISTA C e BASTOS FI, 2015).

A questão das IST entre adolescentes deve ter como foco a necessidade de implantação de estratégias para reduzir os riscos de contágio e transmissão nesta população. A prevenção entre jovens vem, consecutivamente, sendo objetivo de políticas públicas de saúde no Brasil, principalmente, devido à iniciação sexual ocorrer cada vez mais de maneira precoce (OLIVEIRA SHS, et al., 2015). Diante disto, é necessário que o papel do enfermeiro seja efetivo na elaboração de estratégias que alcancem as necessidades do adolescente, contemplando as especificidades da própria etapa de vida (CASTRO EL, et al., 2016).

A questão que impulsionou este estudo foi: qual o nível de conhecimento de estudantes da educação básica sobre infecções sexualmente transmissíveis? Desta forma e na perspectiva de coleta de dados sobre o conhecimento e opinião de jovens e adolescentes no que diz respeito às IST, a pesquisa se propõe verificar o conhecimento e opinião de estudantes da educação básica sobre infecções sexualmente transmissíveis em uma escola pública em um município do estado do Pará.

\section{MÉTODOS}

Trata-se de uma pesquisa transversal exploratória com abordagem quantitativa, realizada em uma Escola Estadual de Ensino Médio, localizada em um Município do estado do Pará, no período de setembro a outubro de 2018. A escolha da Escola se deu por considerarmos uma escola centralizada e por receber alunos da zona ribeirinha, urbana e do campo, onde, possibilitaria uma visão do nível de conhecimento acerca da temática diferenciada devido ao local onde mora e ao período em que se encontram matriculados. 
A amostragem inicial seria de todos os estudantes matriculados do $1^{\circ}$ ao $3^{\circ}$ ano do ensino médio, totalizando 500 alunos com idades entre 13 a 18 anos. Porém houve uma perda da amostra, que passou a contar com 300 indivíduos. A perda da amostragem se deu devido a um número considerável de estudantes não se enquadrarem na faixa etária pesquisada, a não autorização por parte dos responsáveis, 0 preenchimento incompleto do questionário, afastamentos por motivos de doença dentre outros contribuíram para a perda no número da amostra. Como critérios de inclusão foram considerados estudantes com faixa etária de 13 a 18 anos, devidamente matriculados nos turnos matutino, vespertino e noturno, de ambos os gêneros e que os responsáveis autorizassem a participação no estudo.

Os critérios de inclusão foram: os alunos que não estavam matriculados regularmente nos turnos matutino, vespertino e noturno os que possuíam idade abaixo ou acima de 18 anos, que não assinaram o TCLE e o Termo de Assentimento e o que não preencheram ao questionário corretamente.

Após a elucidação das dúvidas sobre a pesquisa em questão, foi fornecido o Termo de Consentimento Livre e Esclarecido (TCLE) contendo devidas orientações para ser analisado e assinado pelos investigados maiores de idade e para os menores levarem para os responsáveis analisarem e autorizarem a participação no estudo.

Foi utilizado como instrumento de coleta de dados um questionário estruturado contendo perguntas fechadas, construído pelos próprios pesquisadores para o alcance do objetivo proposto pelo estudo e aplicado in loco. Na aplicação do questionário foi realizado abordagem em sala de aula após consentimento do professor do horário explicitando o objetivo e a metodologia da pesquisa.

O questionário foi autoaplicável composto por três seções obedecendo à seguinte ordem: 1. Aspectos sociais dos jovens (Faixa etária, Sexo, Raça, Procedência, Religião); 2. Perguntas relacionadas a vida sexual e IST (Idade da primeira relação sexual? Usou camisinha na sua primeira relação sexual?); 3. Conhecimento, prevenção, sinais e sintomas sobre IST e fontes de informação (doenças que se transmite através da relação sexual, de que forma você acha que uma pessoa pode pegar IST? Se já teve orientação sobre sexualidade e orientação sobre IST/AIDS?).

A análise dos dados foi feita através de estatística descritiva simples, onde utilizou-se a planilha eletrônica do Microsoft Excel®, para a análise descritiva com frequências absolutas e relativas e a média das variáveis.

Para procedimento de análise de dados considerou-se como desvio amostral uma margem de $5 \%$, com um nível de confiança de $95 \%$, estabelecendo-se como amostra de $n=500$ alunos, que contabilizou $71,3 \%$ de toda amostra inicial, sendo 100 alunos do primeiro, 100 do segundo e 100 do terceiro ano do ensino médio. Para o desfecho de cálculo amostral foi utilizada uma amostragem aleatória sistemática sobre variáveis categóricas, utilizando-se a seguinte fórmula:

$$
\frac{7: n=N \cdot \text { Z2. p. }(1-p)}{\text { Z 2.p. }(1-p)+\text { e2. }(N-1)}
$$

Onde: $\mathrm{n}$ - amostra calculada; $\mathrm{N}$ - população; $\mathrm{Z}$ - variável normal padronizada associada ao nível de confiança; $p$ - verdadeira probabilidade do evento e, e - erro amostral.

Este estudo foi apreciado pelo Comitê de Ética em Pesquisa da Universidade da Amazônia (UNAMA), sob o parecer CAAE: 52692016.2.000.5173, de 21 de março de 2016.

\section{RESULTADOS}

A amostra foi composta por 300 estudantes que estudavam em uma Escola Estadual de Ensino Médio, localizada em município do estado do Pará. Quanto à caracterização da amostra, 199 (69,23\%) eram do sexo feminino e $101(30,77 \%)$ do sexo masculino, com idade média de $17( \pm 2,69)$ anos. Quanto à religião, 104 (36,36\%) alunos eram católicos, $112(39,17 \%)$ evangélicos, 84 (24,47\%) não informaram. Quanto ao estado civil, $170(59,44 \%)$ eram casados e $130(40,56 \%)$ solteiros. Quanto aos dados ocupacionais, 240 (77,62\%) 
estudavam e 60 (22,38\%) estudavam e trabalhavam. Sobre composição familiar, $180(60,14 \%)$ responderam que possuíam família nuclear e 120 (39,86\%) não informaram.

No que diz respeito sobre os jovens já terem tido a primeira relação sexual $186(62 \%)$ informaram que sim e 114 (38\%) não (Gráfico 1).

Gráfico 1 - Porcentagem dos alunos que já tiveram a primeira experiência sexual em uma escola estadual de ensino médio em um município do estado do Pará.

\section{Experiência sexual ?}

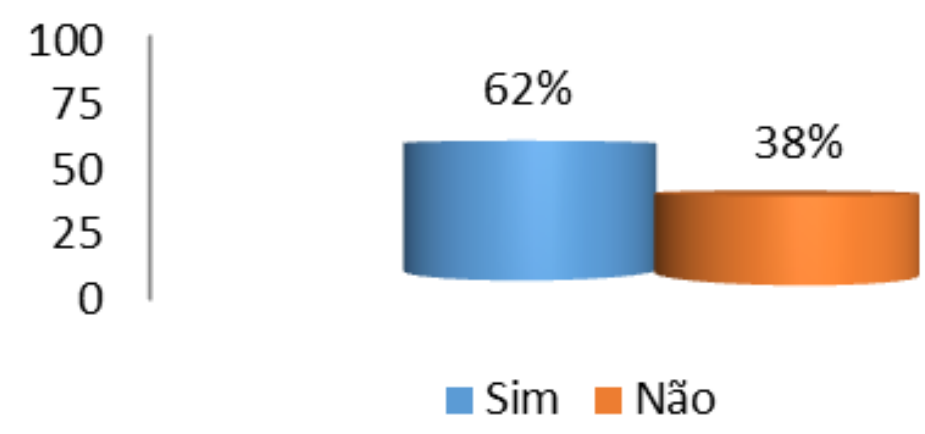

Fonte: Wanzeler ELF, et al., 2020.

Quando questionados sobre a faixa etária em que realizaram a primeira relação, 7 (4,9\%) informam ter realizado antes dos 10 anos de idade e 37\% não informaram (Gráfico 2).

Gráfico 2 - Porcentagem da faixa etária dos alunos quando realizaram a primeira relação sexual. Em um município do estado do Pará, 2018.

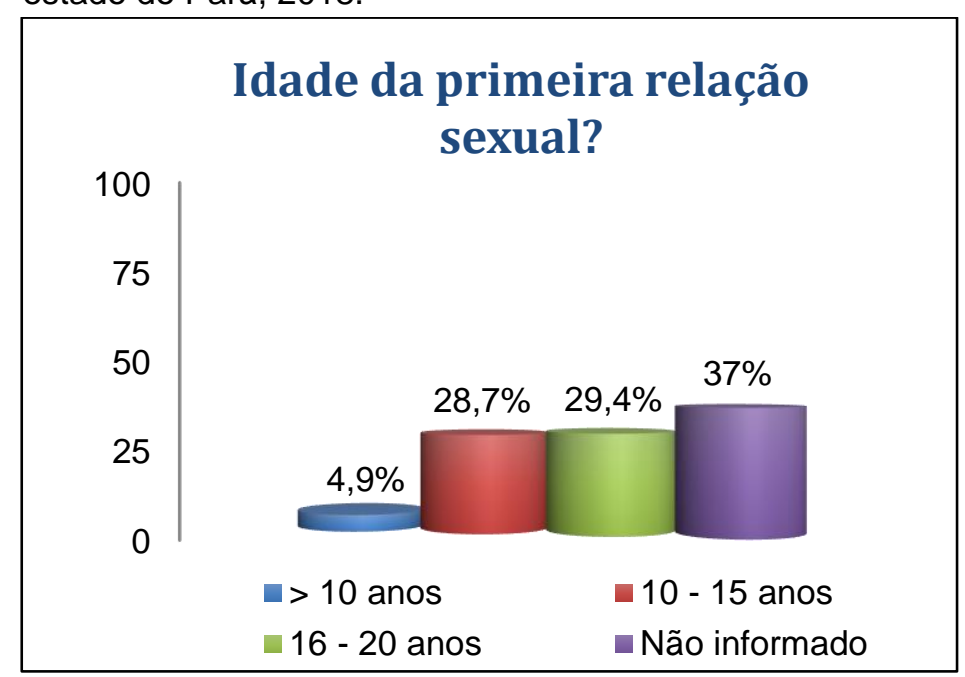

Fonte: Wanzeler ELF, et al., 2020.

Em relação ao uso de preservativo na primeira relação sexual, $92(31,8 \%)$ informaram que usaram, 92 $(31,8 \%)$ não usaram e $116(36,4 \%)$ não informaram. Quando questionados sobre o(s) motivo(s) que os levaram a usarem preservativo na primeira relação sexual $79(26,6 \%)$ disseram para evitar IST e a gravidez e $160(55,2 \%)$ não informaram o motivo. Dentre os estudantes entrevistados, $40(15,4 \%)$ informaram que não usaram preservativos porque confiavam em seus parceiros, $27(9,1 \%)$ afirmaram que não tinham 0 preservativo na hora e $3(0,7 \%)$ informou que não usou devido ao custo ser alto. 
Questionou-se também qual era o relacionamento dos estudantes com a pessoa no período que aconteceu a primeira relação sexual, $180(60,0 \%)$ disseram que foi alguém da própria família ou do convívio familiar e $120(40,0 \%)$ não informaram.

No que diz respeito ao conhecimento dos estudantes sobre as doenças que podem ser transmitidas durante a relação sexual desprotegida (sem o uso de preservativo), $252(82,0 \%)$ dos estudantes responderam que se transmitem doenças infectocontagiosa sistêmica, $2(2,0 \%)$ disseram Varicela, $9(3,0 \%)$ responderam dengue, 9 (3,0\%) informaram Caxumba e 24 (8,0 \%) acharam que nenhuma doença anterior transmite.

Quando questionados sobre quem os estudantes costumam esclarecer suas dúvidas sobre ISTs/ AIDS, $96(32,0 \%)$ procuram a mãe, $63(21,0 \%)$ os livros ou internet, $57(19,0 \%)$ os profissionais da área da saúde, $33(11,0 \%)$ o pai, $30(10,0 \%)$ o namorado, $12(4,0 \%)$ disseram os amigos e $9(3,0 \%)$ o professor. Quanto orientações sobre sexualidade, $114(38,0 \%)$ responderam que já tiveram na escola, $90(30,0 \%)$ com familiares, $60(20,0 \%)$ no posto de saúde e $36(12,0 \%)$ responderam que nunca tiveram.

Confirma-se pelos dados apresentados acima, que muitos jovens sentem dúvidas, sobre a forma de transmissão, ressaltando que percentuais altos indicam a falta de conhecimentos de muitos, e de informações repassadas erroneamente. Dentre os estudantes entrevistados, $222(74,0 \%)$ responderam que se apresentassem alguma IST contaria para seu parceiro, porém 49 (16,0\%) disseram que não contariam e 29 $(9,1 \%)$ não informaram. Quando questionados se já apresentaram alguma IST, $3(1,0 \%)$ disseram que sim, $282(94,0 \%)$ não e $15(5,0 \%)$ não informaram.

\section{DISCUSSÃO}

Sabe-se que o tema é amplamente abordado nas literaturas, haja vista que o perfil epidemiológico de adolescentes em diversos estudos é bastante discutido, devido ao alto risco de contaminação as IST/HIV/AIDS, nesta faixa etária.

Com o resultado final, percebe-se que os jovens selecionados neste desenho vêm se relacionando sexualmente cada vez mais precocemente, que com ( $>10$ anos) $4,9 \%$ dos jovens já teriam mantido sua primeira relação sexual e que um percentual considerável $28,7 \%$ tiveram sua primeira relação sexual, na faixa etária de 10 a 15 anos. Confirmam-se em outros estudos essas informações, pois segundo Silva ADSN, et al. (2015) O início da vida sexual precocemente é uma prática de risco que expõe o adolescente a situações de maior probabilidade, devido aos números de parceiros sexuais nessa fase da vida ou até mesmo para aqueles com relações monogâmicas estáveis, visto que quanto maior o número de parceiros sexuais, maior probabilidade de contágio já que a exposição às ISTs tendem para um aumento significativo(CUNHA NCD, et al., 2017).

Com relação ao credo religioso, a constatação da maioria $(75,53 \%)$ frequentar alguma organização religiosa tem seu significado importante. Visto que valores e crenças, entre eles a religião, compõem elementos que podem interferir na percepção de vulnerabilidade às IST, sobretudo na adoção de métodos de sexo seguro, como é o caso da utilização de preservativos, além de valores relacionados ao casamento e quantidade de parceiros (GUANILO MCDLT, et al., 2014).

No tocante ao estado civil desses jovens, o estudo diverge dos achados na literatura. Uma investigação transversal, com 120 participantes, realizado na cidade de João Pessoa/PB, Brasil, identificou que 62,4\% vivem sem companheiro (GUANILO MCDLT, et al., 2014).

A este respeito, enquanto um estudo aponta que adolescentes e jovens solteiros são mais inclinados para relacionamentos com múltiplos parceiros, aumentando, desta maneira as chances de infecção pelas ISTs, outros demonstram que pessoas casadas ou com união estável são mais propensas a contraírem infecções (PEREIRA BS, et al., 2014; AMARAL RDS, 2016).

Esse dado merece destaque, pois demonstra o impacto que as características sociodemográficas, como o estado civil, podem ter na expansão das IST entre determinados grupos, evidenciando assim, a influência do contexto social sobre a forma como os indivíduos expõem-se a este problema. Dessa forma, o estado civil, 
constitui-se aspecto de extrema relevância para o planejamento e a execução de ações que visem redução de vulnerabilidades as infecções sexualmente transmissíveis bem como ao HIV/AIDS.

Cercando todas as questões que estão relacionadas à suscetibilidade dos jovens da determinada escola pesquisada, questionou-se sobre a composição familiar desses estudantes, a maioria possuía uma família nuclear (60,14\%), que é constituída por pai mãe e filhos. Uma revisão sistemática, com o objetivo de avaliar a associação entre práticas parentais e o comportamento sexual, mostrou que adolescentes que estão em uma estrutura familiar constituída de pai e mãe ainda são vulneráveis em frente a impotência dos pais para atuar na educação sexual dos filhos (ARAGÃO JDS, et al., 2016; GASPAR J, et al., 2015). Isso acontece devido às dificuldades encontradas em abordar o assunto decorrente da falta de informação ou por vergonha (COSTA MIF, 2017).

Relacionou-se o não uso de preservativo na primeira relação sexual com o risco de contrair as IST e HIV. A maioria $(36,4 \%)$ dos entrevistados não lembrou ou não quiseram informar se usaram ou não o preservativo na primeira relação sexual, e que o percentual de pessoas que usaram e as que não usaram foram iguais. Corroborando com esse achado outro estudo revelou que os adolescentes tendem a não usar preservativo no início de sua vida sexual, sendo os principais motivos não gostar de usá-los, confiar no parceiro e a imprevisibilidade das relações sexuais, além de definirem esse tipo de relação como casual (GUANILO MCDLT, et al., 2014).

No que diz respeito a pergunta como às doenças podem ser transmitidas durante a relação sexual desprotegida, constatou-se que os estudantes possuem conhecimento a respeito das doenças, pois a maioria $(81,94 \%)$ respondeu ao questionário e opinaram em Doenças Infectocontagiosa Sistêmica. O conhecimento dos adolescentes sobre as IST vem sendo amplamente abordado em diferentes estudos, nos quais podem ser observados resultados favoráveis ao conhecimento dos mesmos sobre esta temática ou contrária, apontando desconhecimento sobre esse assunto (SCHERMANN LB, et al., 2014; AMARAL RDS, 2016).

Sabe-se que o conhecimento é um importante instrumento de prevenção às IST (CASTRO EL, et al., 2016). Em estudo investigativo, que avaliou o conhecimento de adolescentes sobre a contaminação/transmissão das IST, a AIDS foi a mais reconhecida pelos jovens e o ato sexual sem preservativo a forma de transmissão mais conhecida (SCHERMANN LB, et al., 2014). Os escolares, de um modo geral, demonstram conhecer o que é uma IST, que é adquirida através da relação sexual, e a necessidade do uso do preservativo como uma forma de proteção (SIQUEIRA LOA, 2014).

O único meio de evitar a infecção de IST é a mudança de comportamento de risco, por meio de ações de prevenção, como observado no resultado do questionário: $81,8 \%$ dos estudantes afirmaram que para se proteger deve-se usar preservativo nas relações sexuais. A referência quanto ao uso de preservativos indicado pelos adolescentes como uma forma fundamental na prevenção de IST indicou a importância desse método o que é corroborado por estudos que referem que esse é, indubitavelmente, o método contraceptivo mais conhecido pelos adolescentes (CASTRO EL, et al., 2016; COSTA MIF, 2017).

Pesquisas sobre o comportamento sexual de adolescentes revelam que a proporção de jovens que usam preservativos nas relações sexuais aumentou, contudo, a camisinha ainda não é utilizada por todos e nem em todos os atos sexuais (AMARAL RDS, 2016). Percebe-se que ambiente escolar é bastante adequado para se trabalhar conhecimentos, mudança de comportamento e habilidades, considerando-se que o adolescente permanece boa parte do seu tempo dentro da escola (BATISTA C e BASTOS FI, 2015).

Os estudantes quando questionados a quem procuram para cessar suas dúvidas referente a IST/AIDS a maioria $(32,2 \%)$ relataram a orientação das mães, seguido por pesquisas em livros e internet $(21,7 \%)$, o que reflete a existência de uma confiança no meio familiar. É notório que, cada vez mais, os jovens vêm preocupando-se com a busca por informações sobre as IST e suas formas de prevenção, no entanto nem sempre é no âmbito familiar ou escolar que estes adolescentes encontram as respostas para as questões levantadas (ARAGÃO JDS, et al., 2016; MOREIRA MGF, 2017; SILVA MCDS, 2016). Os pais são exemplos para seus filhos, transmitem valores sendo a primeira referência, porém quando o assunto é sexualidade, muitos pais ou até mesmo os jovens não compartilham suas experiências e dúvidas, ficando o jovem exposto à vulnerabilidade (COSTA TDSD, et al., 2017). 
Em um outro estudo a maioria dos adolescentes afirmaram obter informações sobre prevenção de IST/HIV por meio da televisão e da escola (CHAVES ACP, et al., 2014), que se mostraram como as principais fontes de informação dos adolescentes sobre AIDS. A televisão exerce um importante papel na divulgação de informações sobre modos de transmissão do HIV e de prevenção, já que esta é de fácil acesso entre a população jovem, juntamente com a escola onde acontecem palestras e trabalhos voltados para essa temática (CAMPOS CGAPD, et al., 2014; RODRIGUES MO, et al., 2015).

De acordo com os resultados, observa-se que eles procuram pessoas mais próximas de si e que possam confiar. A adolescência é caracterizada pela vulnerabilidade decorrente das características da própria idade, da falta de habilidades para a tomada de decisões, das dificuldades e, por que não dizer, da inexperiência destes jovens ao lidarem com os seus sentimentos e com os sentimentos dos outros, bem como da responsabilidade nem sempre existente ao se envolverem em relacionamentos afetivos e sexuais (PEREIRA BS, et al., 2014; SIQUEIRA LOA, 2014).

Questionou-se também qual era o relacionamento dos estudantes com a pessoa no período que aconteceu a primeira relação sexual,180 $(60,0 \%)$ disseram que foi alguém da própria família ou do convívio familiar e $120(40,0 \%)$ não informaram. Nas pesquisas realizadas em site de periódicos e após a leitura de inúmeros artigos não se encontrou ensaio que abordassem ou descrevessem a informação acima citada.

$\mathrm{O}$ estudo realizado apresentou limitações importantes quanto à sua população e amostra. A população definida para este estudo, os alunos da Escola Estadual de Ensino Médio José Maria Machado não tiveram autorização por parte dos pais ou responsáveis legais. Isso nos deixa um pouco preocupado com o tabu que ainda existe no âmbito familiar quando se fala em sexualidades e suas nuances. Outra limitação importante se refere ao tamanho da amostra, que ao se apresentar em número reduzido, permite considerar os resultados encontrados apenas para a população em questão.

\section{CONCLUSÃO}

Observa-se nessa reflexão que as situações de vulnerabilidade dos adolescentes e jovens a IST relacionam-se com a não compreensão do uso de preservativos, baixa escolaridade, conhecimentos e informação deficientes sobre IST, fatores culturais e a falta de orientações. São processos desencadeantes que levam os adolescentes a necessitarem de ações educativas contínuas e problematizantes que visem promover a prevenção e uma boa qualidade de vida sexual. Abordar a saúde dos adolescentes nos dias de hoje é de suma relevância, já que essa população é direcionada como um grupo de risco para contaminação com as infecções sexualmente transmissíveis, haja vista, que a adolescência é a fase onde os mesmos passam por mudanças físicas, sociais e psicológicas, gerando conflitos interpessoais e curiosidades a respeito do ato sexual, e junto a ele as novas sensações de sentir prazer, que ocorrem de forma irresponsável e insegura, colocando em risco a sua saúde.

\section{AGRADECIMENTOS}

Agradecemos aos funcionários e alunos da escola objeto de estudo localizada em um Município do estado do Pará, que abraçaram com muito entusiasmo o projeto de pesquisa e contribuíram para que os mesmos tivessem êxito. O nosso muito obrigado.

\section{REFERÊNCIAS}

1. AMARAL RDS. Adolescência, Juventude e HIV/Aids: estudo de fatores associados, Dissertação (Mestrado em Gestão de Programas e Serviços de Saúde) - Programa de Pós-graduação em Gestão de Programas e Serviços de Saúde, Universidade Ceuma, São Luís, 2016; 85 p.

2. ARAGÃO JDS, et al. Vulnerability associated with sexually transmitted infections in physically disabled people. Ciencia \& saude coletiva, 2016; 21(10), 3143-3152.

3. BATISTA C, BASTOS FI. Redes sociais e difusão da AIDS no Brasil. Boletín de la Oficina Sanitaria Panamericana 121:11-44, 2015. 
4. CAMPOS CGAPD, et al. A vulnerabilidade ao HIV em adolescentes: estudo retrospectivo em um centro de testagem e aconselhamento. Revista Mineira de Enfermagem, 2014; 18(2), 310-319.

5. CARNEIRO RF, et al. Educação sexual na adolescência: uma abordagem no contexto escolar. SANARE-Revista de Políticas Públicas, 2015; 14(1).

6. CASTRO EL, et al. O conhecimento e o ensino sobre doenças sexualmente transmissíveis entre universitários. Ciência \& Saúde Coletiva, 2016; 21: 1975-1984.

7. CHAVES ACP, et al. Conhecimentos e atitudes de adolescentes de uma escola pública sobre a transmissão sexual do HIV. Revista Brasileira de Enfermagem, 2014; 67(1).

8. COSTA MIF. Adolescentes em situação de pobreza: resiliência e vulnerabilidade às IST/HIV/AIDS. 2017.

9. COSTA TDSD, et al. Escola, sexualidade, práticas sexuais e vulnerabilidades para as infecções sexualmente transmissíveis (ist). Revista interdisciplinar de ensino, pesquisa e extensão, 2017; 4(1).

10. CUNHA NCD, et al. Pesquisa sobre sexualidade e gravidez na adolescência: uma reflexão acerca da importância da articulação do conhecimento através da participação ativa dos estudantes. VITTALLE-Revista de Ciências da Saúde, 2017; 29(2), 11-22.

11. FERREIRA J. Vídeo e Educação. Porto Alegre: Artes Médicas, 2015.

12. GASPAR J, et al. Sociodemographic and clinical factors of women with HPV and their association with HIV. Rev. latinoam. enferm. 2015; 23(1):74-81.

13. GOMES NCRC, et al. Prevalence and factors associated with syphilis in a Reference Center. Revista da Sociedade Brasileira de Medicina Tropical, 2017; 50(1), 27-34.

14. GUANILO MCDLT, et al. Assessing the vulnerability of women to sexually transmitted diseases STDS/HIV: construction and validation of markers. Revista da Escola de Enfermagem da USP, 2014; 48(SPE), 152-159.

15. MELO GCD. Saúde sexual de pessoas vivendo com HIV/AIDS sob a ótica da promoção da saúde, 2015.

16. MOREIRA MGF. O direito de participação das crianças nas ações de regulação das responsabilidades parentais (Doctoral dissertation), 2017.

17. OLIVEIRA SHS, et al. Adolescentes e AIDS: Fatores que influenciam a intenção de uso do preservativo. DST-J bras Doenças Sex Transm 2015; 17(1): 32-38.

18. PEREIRA BS, et al. Fatores associados à infecção pelo HIV/AIDS entre adolescentes e adultos jovens matriculados em Centro de Testagem e Aconselhamento no Estado da Bahia. Ciênc Saúde Colet. 2014;19(3):747-58.

19. RODRIGUES MO, et al. Conhecimento dos adolescentes de uma escola da rede pública sobre as principais doenças sexualmente transmissíveis. Revista de Enfermagem do Centro-Oeste Mineiro, 2015.

20. SANTOS JOPD. Objetivos Estratégicos Do Desporto Escolar E A Sua Aplicação No Contexto Real De PráticaPerceção Da Comunidade Escolar, 2017.

21. SCHERMANN LB, et al. Estresse em adolescentes: estudo com escolares de uma cidade do sul do Brasil. Aletheia, $2014 ; 43-44,160-173$.

22. SILVA ADSN, et al. Início da vida sexual em adolescentes escolares: um estudo transversal sobre comportamento sexual de risco em Abaetetuba, Estado do Pará, Brasil. Revista Pan-Amazônica de Saúde, 2015; 6(3), 27-34.

23. SILVA JLGD, et al. Prevalencia de Co-infecções em Pacientes HIV/AIDS na região Noroeste do Rio Grande do Sul. Salão do Conhecimento, 2016, 2(2).

24. SILVA MCDS, et al. Compreensão dos adolescentes sobre a prevenção e transmissão das infecções sexualmente transmissíveis, 2016.

25. SIQUEIRA LOA. Juventudes na cidade: um estudo sobre formação e participação, 2014. 